\title{
EQUAÇÕES QUADRÁTICAS: UMA ABORDAGEM HISTÓRICA ATRAVÉS DE RESOLUÇÕES GEOMÉTRICAS
}

\section{QUADRATIC EQUATIONS: AN HISTORICAL APPROACH THROUGH GEOMETRIC RESOLUTIONS}

Christian Luz Pelissari de Oliveira, Elias de Oliveira Boaventura, Richard Mariano de Souza Silva, Alessandra de \Souza Aguiar Silva, Carla Danieli da Silva Conceição, Natalia Silvéria Medeiros, Eugenia Brunilda Opazo Uribe

Universidade Federal de Mato Grosso do Sul - UFMS, Grupo PET Conexões de Saberes- Matemática, Três Lagoas, MS. Agência de fomento: Programa de Educação Tutorial

E-mail: christian_pelissari@hotmail.

RESUMO - $\quad$ O presente artigo é resultado de um trabalho de pesquisa de alunos do Curso de Licenciatura em Matemática no âmbito do Programa de Educação Tutorial. O objetivo é explorar aspectos da História da Matemática relacionados à resolução geométrica de equações quadráticas, buscando subsídios históricos para complementar o ensino deste tópico nas aulas de Matemática do Ensino Básico, tornando-o mais concreto e interessante para os alunos. O trabalho foi desenvolvido em grupo através de pesquisa bibliográfica e estudo teórico, incluindo seminários, exercícios e interpretações geométricas. Foi explorado o método babilônico, bem como a solução geométrica apresentada pelo árabe Al-Khwarizmi. Concluímos que a abordagem histórica é importante como elemento motivacional e de apoio para o aprendizado, em lugar de basear o ensino em fórmulas prontas e sem significado para o aluno.

Palavras-chave: História da Matemática; Resolução Geométrica de Equações; Equações Quadráticas; Programa de Educação Tutorial.

ABSTRACT - The present article is the result of a research made by Mathematics students in the Tutorial Education Program. The goal is to explore the aspects of Mathematics History related to the geometrical resolution of quadratic equations, searching historical elements to complement the teaching of this topic in Mathematics classes of Elementary School, making it more concrete and interesting for students. The work was developed in group through bibliographic research and theoretical study, including seminars, exercises and

Recebido em: 19/08/2015 Revisado em: 01/09/2015 Aprovado em: 05/09/2015 geometrical interpretations. The Babylonian method was explored, as well as the geometrical solution presented by the arab Al-Khwarizmi. We concluded that the historical approach is important as a motivational element and of support of learning, instead of teaching based on prepared formulas without meaning to the student. 
Keywords: History of Mathematics; Geometrical Resolution of Equations; Quadratic Equations; Tutorial Education Program. 


\section{INTRODUÇÃO}

As Orientações Curriculares para o Ensino Médio (BRASIL. Ministério da Educação, 2006) resaltam a importância da História da Matemática na formação do professor de Matemática, afirmando que: “A História da Matemática pode contribuir também para que o próprio professor compreenda algumas dificuldades dos alunos, que de certa maneira, podem refletir históricas dificuldades presentes também na construção do conhecimento matemático", reafirmando ainda a importância do seu uso em sala de aula: "A utilização da História da Matemática em sala de aula também pode ser vista como um elemento importante no processo de atribuição de significados aos conceitos matemáticos".

O objetivo do presente trabalho é explorar aspectos da História da Matemática relacionados à resolução geométrica de equações quadráticas, buscando subsídios históricos para complementar o ensino deste tópico nas aulas de Matemática do Ensino Básico, tornando-o mais concreto e interessante para os alunos. Assim, após uma breve abordagem histórica, estudaremos algebricamente os métodos utilizados pelos babilônios e geometricamente os métodos utilizados pelos árabes, em particular o matemático Al-Khwarizmi.

\section{METODOLOGIA}

O trabalho foi desenvolvido em grupo através de pesquisa bibliográfica e um estudo teórico que incluiu apresentação de seminários, resolução de exercícios e interpretações geométricas.

\section{RESULTADOS}

\section{ALGUNS ASPECTOS HISTÓRICOS}

Refatti e Bisognin (2005) afirma baseada em textos de História da Matemática que "o conceito de equação quadrática estudado no ensino fundamental tem sua origem na antiguidade. Encontramse registros de matemáticos do Egito, da antiga Babilônia, da Grécia, da Índia, da Arábia e da Europa Medieval sobre problemas referentes a esse tema".

Os matemáticos da antiga Babilônia utilizavam tabletes de argila úmida como material de escrita, que posteriormente eram cozidas em forno até endurecer, conhecidas como tábuas cuneiformes (EVES, 2011), além disso, tinham um sistema de numeração posicional bem desenvolvido, com base 60 (CARVALHO, 2004). Segundo Boyer (1974) “A solução de uma equação quadrática parece ter sido demasiado difícil para os egípcios, mas Neugebauer em 1930 revelou que tais equações tinham sido tratadas eficientemente pelos babilônios em alguns dos mais antigos textos de problemas". Assim, os babilônios apresentavam uma 
álgebra mais desenvolvida do que a Matemática egípcia (CARVALHO, 2004). Um problema encontrado num dos tabletes que aborda uma equação quadrática é "achar o lado de um quadrado se sua área menos seu lado é igual a 870".

Um dos primeiros e mais ilustres matemáticos árabe foi o estudioso Abu Jafar Mohamed ibn Musa Al-Khwarizmi (780-850), um astrônomo do califa de Bagdá. Em seu livro Al-jabr we'l muqabala, cujo título completo é O livro compendioso dos cálculos com al-jabr e al-muqabala, Al-Khwarizmi dá dadas regras para as resoluções de equações do primeiro e do segundo graus (CARVALHO, 2004).

\section{MÉTODOS UTILIZADOS PELOS BABILONICOS}

\section{PARA RESOLVER EQUACCÕES QUADRÁTICAS}

Até os tempos modernos não havia ideia de resolver uma equação quadrática da forma

$$
x^{2}+p x+q=0,
$$

onde $p$ e $q$ são positivos, pois a equação não tem raiz positiva. Por isso as equações quadráticas na antiguidade e na Idade Média, e mesmo no começo do período moderno, foram classificadas em três tipos

$$
\begin{aligned}
& \text { 1. } x^{2}+p x=q \\
& \text { 2. } x^{2}=p x+q \\
& \text { 3. } x^{2}+q=p x
\end{aligned}
$$

Todos esses tipos são encontrados em textos babilônicos (BOYER, 1974). Mas, os babilônios não dispunham de um simbolismo algébrico para escrever estas equações (CARVALHO, 2004), além disso, segundo Refatti e Bisognin (2005), eles desenvolveram uma aproximação algorítmica para resolver problemas envolvendo equações quadráticas que é citada por historiadores matemáticos como uma "receita matemática".

Exemplo 1. Considerando o exemplo citado anteriormente: achar o lado de um quadrado se sua área menos seu lado é igual a 870 . Podemos reescrever ele utilizando a linguagem atual como

$$
x^{2}-x=870 .
$$

A solução proposta pelos babilônios é, - Tome a unidade (coeficiente de $\mathrm{x}$ ): 1 ,

- Divida a unidade em duas partes: $1 / 2$,

- Multiplique $1 / 2$ por $1 / 2: 1 / 4$,

- Some $1 / 4$ a $870: 3481 / 4$,

- obtém-se um quadrado de lado $59 / 2$,

- Some $1 / 2$ a esse lado e obterá 30 , o lado do quadrado procurado.

Para uma equação da forma $x^{2}-p x+q$ $=0$, com $\mathrm{p}$ e q positivos, existe uma raiz positiva dada por $x=\sqrt{\left(\frac{p}{q}\right)^{2}+q}+\frac{p}{2}$,

- algoritmo apresentado pelos babilônios é equivalente a aplicar esta fórmula. Pesquisas recentes e minuciosas sobre a matemática babilônia sugerem que os escribas babilônios chegaram a este 
resultado usando raciocínios geométricos (CARVALHO, 2004).

MÉTODOS UTILIZADOS POR AL-KHWARIZMI PARA RESOLVER EQUACCÕES QUADRÁTICAS

Como os árabes não trabalhavam com números negativos, os coeficientes, bem como as raízes das equações tinham que ser positivos, Al-Khwarizmi utilizou seis casos de equações (REFATTI; BISOGNIN, 2005)

1- Quadrados iguais a raízes $\quad a x^{2}=b x$

2- Quadrados iguais a números $a x^{2}=c$

3- Raízes iguais aos números $\quad b x=c$

4- Quadrados mais raízes iguais a

número $a x^{2}+b x=c$

5- Quadrado mais números iguais a

raízes $\quad a x^{2}+c=b x$

a)

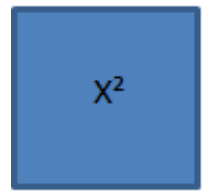

Figura 1.

Fonte: Elaborada pelos autores

Agora, acrescentamos quatro

pequenos quadrados de lado 3 e área 9

(figura 2a). Assim, os quatro quadrados
6- $\quad$ Raízes mais números iguais a

quadrado

$b x+c=a x^{2}$

Em que "quadrado" representa o quadrado da incógnita, "raízes" representa a incógnita e "número" representam os números no nosso sistema atual . Para os três primeiros casos a solução é direta, então AlKhwarizmi trabalhou com os três últimos casos.

Exemplo 2. Consideremos o caso quadrado mais raiz igual número, através da equação $x^{2}+12 x=64$.

Representamos um quadrado como $\mathrm{x}^{2}$ (figura 1a) e acrescentamos ao seu redor quatro retângulos cada um com largura 3 e altura $x$, representando $12 x$. Assim, obtemos uma figura de área $x^{2}+12 x$ ou área igual a 64 (figura 1b).

b)

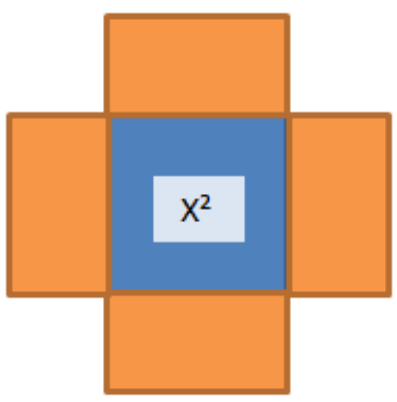

representam uma área igual a 36 . Obtendo assim, um novo quadrado de área $64+36=$ 100 (figura 2b). 
a)

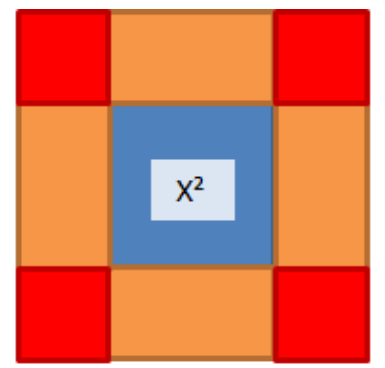

b)

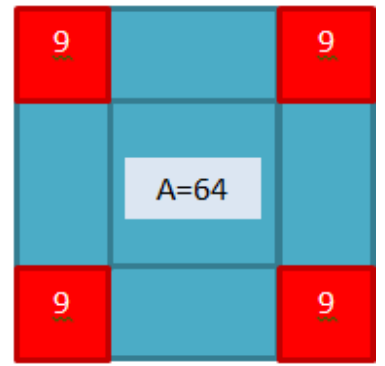

\section{Figura 2.}

Fonte: Elaborada pelos autores

Como a área total do novo quadrado é 100 , temos um quadrado de lado 10 , do qual devemos subtrair $2 \times 3=6$, referente ao lado dos dois quadrados menores (figura 3).

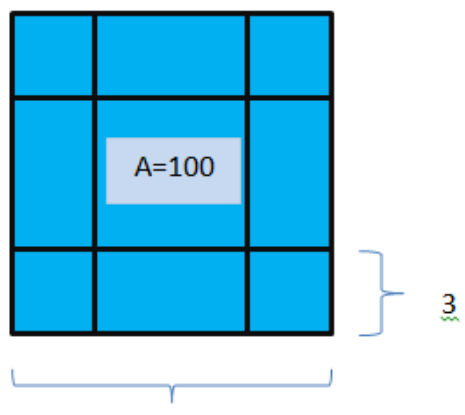

10

\section{Figura 3.}

Fonte: Elaborada pelos autores

Sendo assim $10-6=4$, então o lado do quadrado é 4 ou $x=4$.

\section{Exemplo 3. Consideremos o caso} quadrado mais número igual à raiz, através da equação $x^{2}+21=10 x$.
Representamos um quadrado ACDB como $x^{2}$ (figura 4a) e acrescentamos o retângulo BDHG para representar 21. 
a)

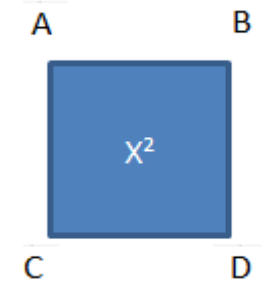

\section{Figura 4.}

Fonte: Elaborada pelos autores

Então a área do retângulo maior corresponde à soma das áreas do quadrado ACDB e do retângulo $B D H G$, isto é, $x^{2}+21,0$ que significa que a área é igual a 10x. Logo o lado $A G$ é igual a 10 e o lado $A C(=G H)$ é igual a x (figura 4b). Prosseguindo, representamos por $E$, o ponto médio do lado $A G$ e, em

a)

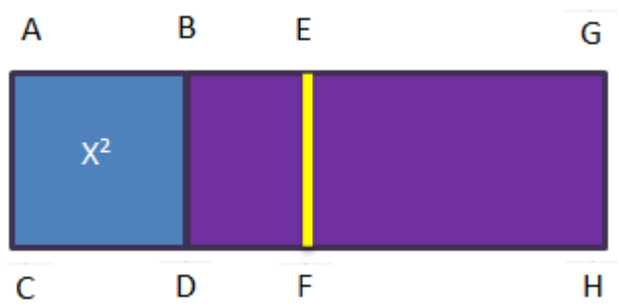

Figura 5.

Fonte: Elaborada pelos autores

Agora completamos os quadrados EKIG e FKJL, de maneira que o quadrado EKIG tem área igual a 25 e o quadrado FKJL tem área igual à $(5-X)^{2}$ (figura 6a). A área do b)

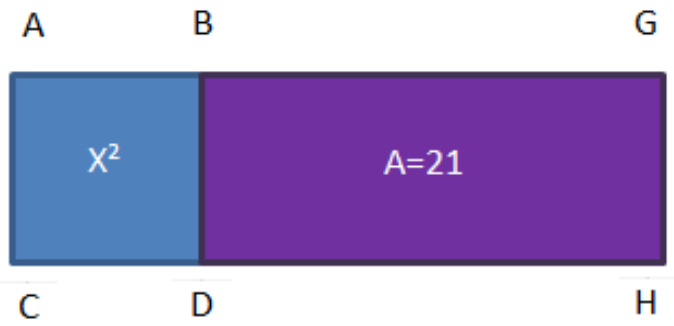

seguida, traçamos EF perpendicular ao lado $\mathrm{CH}$, dividindo assim, o retângulo ACHG em duas partes iguais (figura 5a). Prolongamos $\mathrm{EF}$ até $\mathrm{K}$ de maneira que $\mathrm{EK}=\mathrm{EG}=\mathrm{CF}$ e assim, a medida dos três é 5 . b)

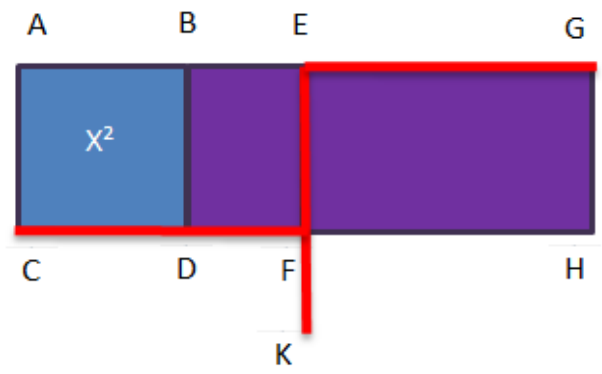

retângulo BDFE é igual à área do retângulo LIH (figura 6b). 
a)

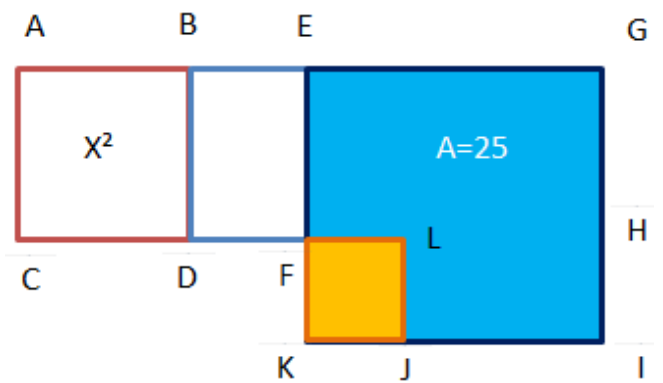

b)

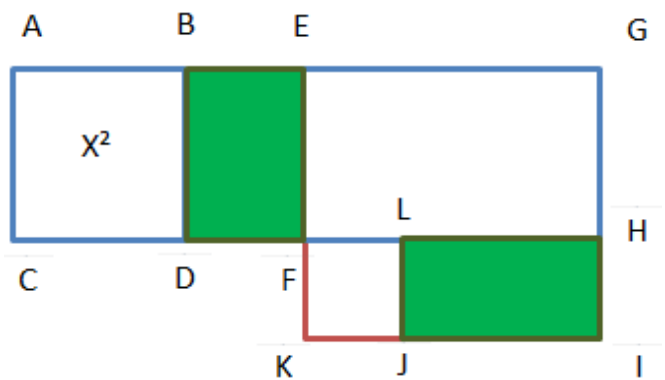

Figura 6.

Fonte: Elaborada pelos autores

Sabendo que o quadrado EKIG tem área igual a 25 e que o polígono formado por EFHG + LJIH tem área igual a 21, podemos concluir que a área do quadrado FKJL é 4 (figura 7a, b). Logo o lado do quadrado FKJL é igual a 2. Como $F K=D F$ e como $C F=5$, temos $C D=C F-D F$, obtendo assim que $C D=5-2=3$ (figura 8 ).

a)

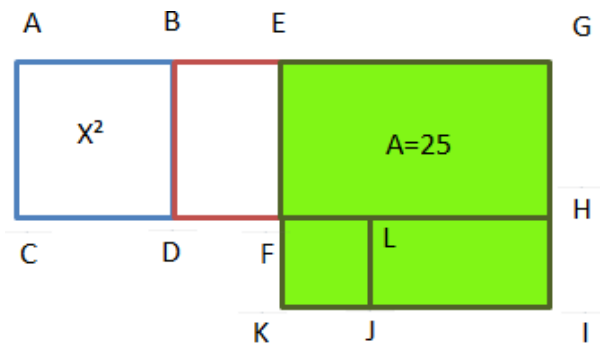

b)

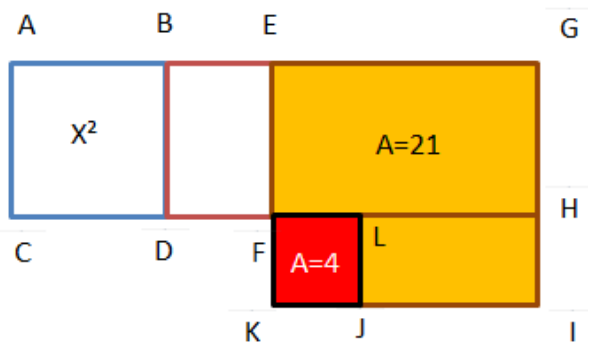

Figura 7.

Fonte: Elaborada pelos autores

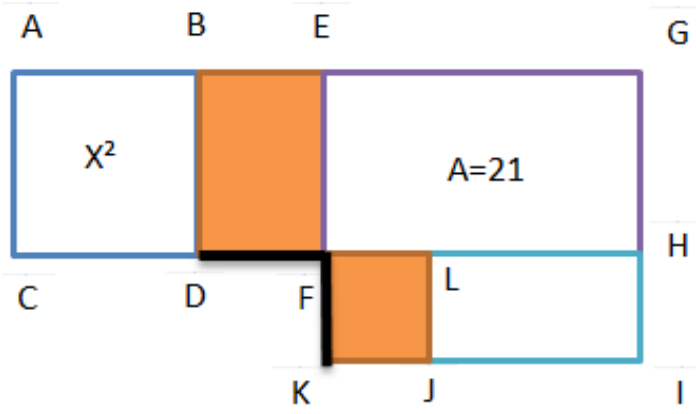

Figura 8.

Fonte: Elaborada pelos autores 


\section{DISCUSSÃO}

Através da resolução de exercícios e seminários apresentados durante 0 desenvolvimento do trabalho foi possível verificar a evolução dos métodos de solução das equações quadráticas ao longo do tempo. Foram analisados todos os tipos de equações de acordo com a classificação utilizada pelos babilônios e pelo árabe AlKhwarizmi, representando as soluções através de justificativas algébricas e geométricas.

\section{CONCLUSÃO}

Através do presente trabalho foi possível verificar que a História da Matemática é uma fonte muito rica de material para o ensino da Matemática e especificamente no caso da Equação quadrática, ela nos proporciona métodos diversificados devido às contribuições feitas por matemáticos de diferentes épocas. Além disso, foi possível observar que a geometria se apresenta como uma valiosa ferramenta para a resolução de diversos problemas algébricos. Assim, combinando métodos geométricos com subsídios históricos, podemos complementar as aulas de Matemática de maneira a torná-las mais interessante para os alunos.

\section{REFERÊNCIAS}

BOYER, C. B. História da matemática. São Paulo: Edgard Blucher, 1974.

BRASIL. Ministério da Educação. Secretaria de Educação Básica. Ciências da natureza, matemática e suas tecnologias. Brasília: Ministério da Educação, 2006. 135 p. (Orientações curriculares para o ensino médio; v. 2).

CARVALHO, J. B. P. F. Revisitando uma velha conhecida - a história da equação do segundo grau. In: BIENAL DA SOCIEDADE BRASILEIRA DE MATEMÁTICA, 2. ANAIS... Salvador, BA, p.1-49, 2004.

EVES, H. Introdução à história da matemática. 5. ed. Campinas: Editora da Unicamp, 2011.

REFATTI, L. R.; BISOGNIN, E. Aspectos históricos e geométricos da equação quadrática. Disciplinarum Scientia. Série Ciências Naturais e Tecnológicas, v. 6, p. 7995, 2005. 